\title{
Summary Report of Working Group 6: Laser-Plasma Acceleration
}

\author{
Wim Leemans*, Michael Downer ${ }^{\dagger}$, and Craig Siders** \\ * LOASIS Program, Lawrence Berkeley National Laboratory, \\ University of California, Berkeley, California 94720 \\ ${ }^{\dagger}$ Departmem of Physics, University of Texas, Austin, Texas 78712 \\ "* Photon Science and Applications Program, National Ignition Facility Directorate, \\ Lawrence Livenmore National Laboratory. Livermore, California 94550
}

\begin{abstract}
A summary is given of presentations and discussions in the Laser-Plasma Icceleration Working Group at the 2006 Advanced Accelerator Concepts Workshop. Presentation highlights include: widespread observation of quasi-monoenergetic clectrons; good agreement betwetn measured and simulated beam properties; the first demonstration of laserplasma accelemtion up to $1 \mathrm{GeV}$; single-shot visualization of laser wakefield structure; new: methuds for measuring $<100$ fs electron bunches; and new methods for "machining" laserplasma accelerator structures. Discussion of future directions includes: developing a rondmap for laser-plasma acceleration beyond $1 \mathrm{GeV}$; a debate over injection and guiding; benchmarking simulations with improved wake diagnostics; petawatt laser technology for future laser-plesma accelerators.
\end{abstract}

Keywords: laser, plasma, accelerator

PACS: $41.75 . \mathrm{Ht}, 41.75 . \mathrm{Jv}, 52.38 . \mathrm{Kd}$

\section{INTRODUCTION}

Working Group 6 (WG 6) focused on acceleration of electrons using laser-driven underdense plasma structures. Prior to the 2006 Advanced Accelerator Concepts Workshop (AAC 06), laser-plasma accelerators had produced intense beams with percent level energy spread, and normalized emittance on the order of $1 \mathrm{~mm}$-mrad at the $100 \mathrm{MeV}$ level [1-3]. This year, WG 6 examined the design and issues surrounding acceleration of electrons up to and beyond the $1 \mathrm{GeV}$ level while continuing to improve emittance and energy spread. It started discussing development of a community roadmap for reaching beam energies on the TeV-scale. A challenge of such magnitude will have to engage the entire international community of accelerator, laser-plasma and laser physicists. Methods to improve efficiency, average current, and reliability of laser-plasma accelerators, and to develop the advanced diagnostic and simulation capabilities that they will demand, were fundamental components of the discussion in Working Group 6.

The working group sessions were well attended (up to 80 attendees per session) with 32 talks ( 7 invited and 25 contributed) and 10 posters. We held a lively joint session with working group 1 on the question "When will simulations catch up with experiments (or vice versa)?" An opening oral session on Tuesday highlighted 5 
invited talks from major experimental groups summarizing latest results, status and plans in the areas of laser guiding ( $\mathrm{S}$. Hooker, Oxford), unguided laser-plasma acceleration (Y. Glinec, LOA), simulations (W. Mori, UCLA), diagnostics (M. Uesaka, U. Tokyo) and laser technology (C. Toth, LBNL). The remaining sessions on Wednesday through Friday featured contributed talks and workshop-style discussions on guiding structures (led by M. Downer), injection of electrons into laser-plasma accelerators (led by W. Leemans), simulations vs. experimental diagnostics (led by $M$. Downer), scaling laws for laser-plasma accelerators (led by C. Siders) and laser technology (led by $\mathrm{C}$. Siders). The group organized itself to identify and discuss the following key questions:

1. To guide or not to guide?

2. How to stage? What is the role of self-trapping in consecutive stages? What is the optimum stage design? Will consecutive stages be different?

3. How stable is a single laser driving beam? Can we develop positron accelerators?

4. What new diagnostics need to be developed? How does one synchronize, align, and couple beams in and out?

5. Do we have the necessary simulations to help design next-generation laserplasma accelerators?

6. What new concepts are emerging to scale up driving lasers? What is a possible $\mathrm{R} \& \mathrm{D}$ path for laser development in the near, medium and long term?

Participants were encouraged to come prepared to address these questions. Emphasis was on interactive, creative discussion.

\section{WORKING GROUP 6 HIGHLIGHTS}

Two years ago, AAC 2004 highlighted reports by groups at Rutherford Appleton Laboratory (RAL)/Imperial College (IC) [1], Lawrence Berkeley National Laboratory (LBNL) [2] and Laboratoire d'Optique Appliquée (LOA) [3] of production of highquality, quasi-monoenergetic beams from 70 to $170 \mathrm{MeV}$ by laser wakefield acceleration (LWFA). This year WG 6 highlighted widespread observation of quasimonoenergetic LWFA beams around the world. In addition to new results from the 3 original groups (discussed below), we heard reports of quasi-monoenergetic beams from Michigan (S. Reed et al.), Taiwan (S.-Y. Chen et al.), Japan (T. Hosokai, M. Kando, T. Reiko and K. Koyama), and the Naval Research Laboratory (NRL, S. Kaganovich et al.). The Michigan experiments revealed systematic trends over a range $1.5<n_{e}<4 \times 10^{19} \mathrm{~cm}^{-3}$ of plasma density. The highest energy ( $300 \mathrm{MeV}$ ) occurred at the lowest density, the highest charge $(0.5 \mathrm{nC})$ at the highest density. The lowest density at which electron injection was obtained was around $1.5 \times 10^{19} \mathrm{~cm}^{-3}$ using 40 TW laser pulses. In Japan, T. Hosokai (U. Tokyo) reported a degree of control over quasi-monoenergetic bunch production by manipulating pre-plasma with an external magnetic field [4], while T. Kando (Advanced Photon Research Center-JAERI) reported quasi-monoenergetic bunch generation with a record low laser intensity of $4 \times 10^{17} \mathrm{~W} / \mathrm{cm}^{2}$, with careful optimization of pre-pulses and laser focus position in the jet. 
Meanwhile, simulations done by the UCLA group have achieved impressive overall agreement with the results of the $2004 \mathrm{RAL} / \mathrm{IC}$, LBNL and LOA experiments. F. Tsung (UCLA) reported that particle-in-cell simulation of the RAL/IC experiments [1] yielded $15 \%$ higher charge and $20 \%$ higher energy than in the experiments with about 3 times higher energy spread. For the LBNL experiments [2], the simulations predicted about $13 \%$ less charge with an energy distribution that was very similar to the experiment. For the LOA experiments [3], simulations predicted about $30 \%$ lower charge than reported for the experiment at $\sim 17 \%$ higher mean energy than in the experiment) with about 2-3 times narrower spread. These results demonstrate that physical understanding is advancing hand-in-hand with experimental progress.

Major new experimental progress by the LBNL and LOA groups provided additional WG 6 highlights. A collaboration between LBNL and Oxford University produced the first report (WG 6 talks by K. Nakamura and B. Nagler) of laser wakefield acceleration up to $1 \mathrm{GeV}$, a major milestone for the field [5]. This groundbreaking experiment used a gas-filled capillary discharge waveguide developed by the Oxford group [6] to guide 40 TW pulses over more than $3 \mathrm{~cm}$ of plasma. A few percent energy spread and milliradian beam divergence were observed at $1 \mathrm{GeV}$ [5]. Stable beam generation was reported at the $0.5 \mathrm{GeV}$ level, where as low as $\sim 12$ TW laser pulses produced bunches with $5.6 \%$ energy spread, $1.6 \mathrm{mrad}$ divergence, $\sim 50 \mathrm{pC}$ from the channel on a regular basis. Meanwhile the LOA group demonstrated a major advance from self-injected to externally injected quasi-monoenergetic LWFA. Selfinjection prevailed at plasma densities $n_{e} \geq 10^{19} \mathrm{~cm}^{-3}$, but at $n_{e}=7.5 \times 10^{18} \mathrm{~cm}^{-3}$, below this self-injection threshold, the LOA group observed $>100 \mathrm{MeV}$ quasimonoenergetic bunches when a counter-propagating "colliding" injection laser pulse was introduced with identical polarization to the drive laser. No injection was observed when using orthogonal polarizations for drive and colliding laser pulse suggesting that the beat-wave produced by the two colliding beams is responsible for the injection mechanism. The colliding-pulse injection concept had been introduced theoretically by E. Esarey [7] at the 1996 AAC workshop followed by several subsequent publications from the LBNL group on different colliding pulse geometries (e.g., [8]) including preliminary experimental results reported at AAC2004 [9] showing injected charge enhancement. The use of laser guiding structures over cmscale distances, laser triggered injection and stabilization of injection with control of pre-plasma, better control of the laser and plasma parameters for improved performance all signal a major new trend toward controlled acceleration in the laserplasma accelerator field.

Major advances in wakefield and bunch diagnostics were reported in WG 6. N. Matlis (U. Texas) showed "snapshots" of laser wakefjelds [10] obtained using frequency-domain holography $[11,12]$ in a collaboration with a U. Michigan group. The snapshots captured evolution of multiple wake periods, detected structure variations as laser-plasma parameters changed, and resolved wavefront curvature, features never previously observed, except in simulations. Such real-time visualization can potentially enable optimization and feedback control of laser (or charged-particle-driven) plasma accelerators, and provides an earlier experimental point of contact with simulation and theory than electron beam properties. Meanwhile M. Uesaka (U. Tokyo) and C. Geddes (LBNL) highlighted advances in temporal 
characterization of electron bunches from laser-plasma accelerators. Since LWFAs produce femtosecond duration bunches, new techniques are required. Uesaka discussed measurement of $<100 \mathrm{fs}$ bunch durations using spectral analysis of coherent transition radiation (CTR) generated in thin Ti foils detected in multiple shots by an IR bolometer with different filters, or in a single shot by a multi-channel polychromator array [13]. Geddes highlighted measurements of $<50$ fs LWFA bunches based on terahertz CTR emitted at the plasma-vacuum exit boundary of the LWFA gas jet $[14,15]$. Terahertz radiation was detected electro-optically in $\mathrm{ZnTe}$ or $\mathrm{GaAs}$ in a single-shot. V. Yakimenko (Brookhaven) described measurements of $30 \mathrm{fs}$ microbunches based on measurements of CTR harmonics. Finally W. Kimura described characterization of chicane bunch compression at Brookhaven, while I. Blumenfeld presented various bunch length measurements related to the E167 experiment at SLAC. A common theme is that bunch duration measurement in the low femtosecond regime appears within reach.

Reports of "laser-machined" plasma structures by groups at U. Taiwan and U. Maryland signaled another important development for laser-plasma acceleration. At previous AAC workshops, the production of transversely-engineered laser-generatedplasma waveguides [1'6-18] had heen a prominent theme. The Taiwan and Maryland groups have now developed methods for longitudinally modulating plasma structures. The Taiwan group passed the machining laser pulse through an amplitude-modulating structure (e.g., a knife-edge or liquid crystal modulator), then imaged it transversely onto the plasma [19]. The Maryland group employed a mask at the base of a conical axicon lens, then brought the cylindrically symmetric machining beam to a modulated line focus. In both cases, light regions of the image ionized and heated the plasma, causing it to expand locally to low density. S. Y. Chen and C.T. Hsieh (Taiwan) used a sharp-edged "tomography" laser to create a sharp density downramp in the plasma. At previous AAC workshops, wavebreaking induced by an intense laser pulse traversing such a downramp had been proposed theoretically as an electron injection source for laser-plasma accelerators [20-22]. The Taiwan experiments have now realized this concept in the laboratory [23]. Monoenergetic electrons can be produced, depending on position of the down-ramp. Researchers at both Taiwan and Maryland (represented by B. Layer and A. York) described laser machining of variable-period corrugated waveguides in a clustered jet. Quasi-phase-matched high-order harmonic generation was realized in such a waveguide [24].

These highlights constitute only a sampling of the presentations within WG 6. For the complete picture we refer the reader to the many contributed papers within this Proceedings.

\section{PROSPECTS FOR 2008 AND BEYOND}

Where are laser-plasma accelerators headed? Will they use guiding and external injection? How will they be diagnosed? What lasers will drive them? These are some of the questions that dominated the workshop-style discussions of WG 6 . Some of them could not yet be resolved. Nevertheless, the outlines of a roadmap are emerging. 
TABLE 1. Parameter designs for future GeV to TeV laser-plasma accelerators based on 3D particle-in-cell simulations by the UCLA group. Cases with (without) preformed channels labeled with superscript a (b).

\begin{tabular}{|c|c|c|c|c|c|c|}
\hline $\begin{array}{l}\text { Laser } \\
\text { Power } \\
\text { (PW) }\end{array}$ & $\begin{array}{c}\text { Pulse } \\
\text { duration } \\
\text { (fs) }\end{array}$ & $\begin{array}{c}\text { Plasma } \\
\text { density }\left(\mathrm{cm}^{-3}\right)\end{array}$ & $\begin{array}{c}\text { Spot } \\
\text { size } \\
(\mu \mathrm{m})\end{array}$ & $\begin{array}{c}\text { Int" } \\
\text { Iength } \\
\text { (m) }\end{array}$ & $\begin{array}{c}\text { Electron } \\
\text { charge } \\
\text { (nC) }\end{array}$ & $\begin{array}{c}\text { Energy } \\
\text { gain } \\
(\mathrm{GeV}) \\
\end{array}$ \\
\hline $0.02^{\circ}$ & 30 & $1 \times 10^{18}$ & 14 & 0.016 & 0.18 & 0.99 \\
\hline $0.04^{\dagger}$ & 30 & $1.5 \times 10^{18}$ & 14 & 0.011 & 0.25 & 0.95 \\
\hline $0.10^{\circ *}$ & 30 & $2.0 \times 10^{1 \mathrm{ln}}$ & 15 & 0.009 & 0.4 & 1.06 \\
\hline $0.20^{\circ}$ & 100 & $1.0 \times 10^{17}$ & 45 & 0.52 & 0.57 & 9.9 \\
\hline $2.0^{6}$ & 100 & $3.0 \times 10^{17}$ & 47 & 0.18 & 1.8 & 10.2 \\
\hline $2.0^{\circ}$ & 310 & $1.0 \times 10^{16}$ & 140 & 16.3 & 1.8 & 99 \\
\hline $40^{6}$ & 330 & $4.0 \times 10^{16}$ & 146 & 4.2 & 8 & 106 \\
\hline $20^{3}$ & 1000 & $1.0 \times 10^{15}$ & 450 & 500 & 5.7 & 999 \\
\hline $1000^{\mathrm{b}}$ & 1000 & $6.5 \times 10^{15}$ & 450 & 82 & 40 & 1040 \\
\hline
\end{tabular}

channel-guided, extemally injected, $P / P_{c}=0.7$

${ }^{\dagger}$ channel-guided, self-injected, $P / P_{c}=2$

" self-guided, self-injected

Increasingly fast, powerful simulations are drawing much of the roadmap. Using results from the 3D particle-in-cell simulation code OSIRIS, as well as QuickPIC, W. Lu, F. Tsung, M. Tsoufraz and W. B. Mori of UCLA laid out a parameter design table for future laser-plasma accelerators that produce electron bunches ranging from 1 $\mathrm{GeV}$ to $1 \mathrm{TeV}$ in energy. They required that LWFAs, to be useful, should have a stable plasma structure, dephasing length equal to pump depletion length for optimum efficiency, and a reasonable balance between energy extraction and beam quality. A few examples of entries from their extensive table are shown in Table 1. While readers should consult the WG 1 summary and proceedings and other published papers of these authors for full details, a few general trends emerge. Driving laser pulse energy must increase from tens of TW to tens of PW. Plasma density must correspondingly decrease from the range $10^{18}<n_{e}<10^{19} \mathrm{~cm}^{-3}$ prevalent in recent experiments down to the range $10^{15}<n_{c}<10^{16} \mathrm{~cm}^{-3}$. As a result, optimum driving pulse duration will increase from $\sim 30 \mathrm{fs}$ to several hundred $\mathrm{fs}$, optimum focused beam waist from $\sim 15 \mu \mathrm{m}$ to several hundred $\mu \mathrm{m}$, and optimum interaction length from $\sim 1 \mathrm{~cm}$ to tens of meters. The UCLA table included viable designs using both channel and self-guiding, and both external and self-injection. Thus it appears that researchers will continue to pursue a variety of approaches to LWFA for the foreseeable future.

For researchers lacking access to powerful simulations and/or willing to settle for a less rigorous approach to LWFA design, C. Siders (LLNL) argued that a LWFA design study based on simple scaling laws, worked out ten years ago at the International Workshop for 2nd Generation Plasma Accelerators in Kardamyli, Greece [25], can still provide rough guidance today. Figure 1 reproduces one of the parametric plots from that original study, with recent and projected future LWFA results super-imposed. The UCLA group cautioned that pulse parameters such as peak power that evolve during an experiment or simulation are assumed static in such simple estimates. More general scaling laws that take such evolution into account are included in the UCLA study. 


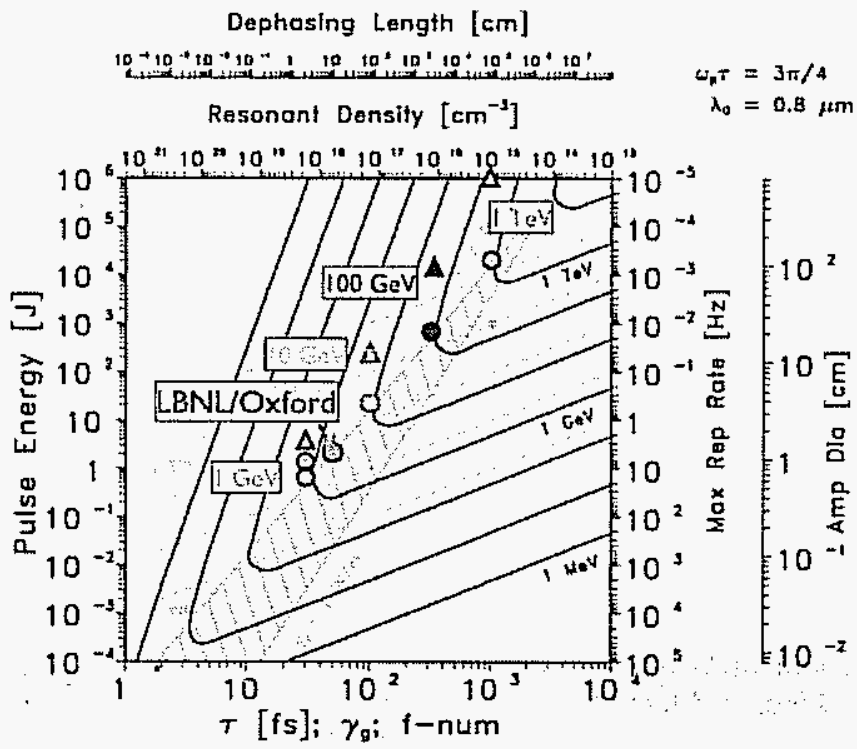

FIGURE 1: Parametric plot for laser-plasma accelerator design based on simple scaling laws [25], with recent (LBNL/Oxford) results [5] and projected future experimental milestones for self-guided (triangles) and guided (circles) LWFA super-imposed. The bottom horizontal axis is laser pulse duration, which is proportional to $\gamma_{\mathrm{g}}$ for Gaussian laser pulses. The top horizontal axis is dephasing length and resonant plasma density. Left vertical axis is pulse energy, right vertical axis shows laser system repetition rate and minimum final amplifier diameter to stay below damage fluence of $2 \mathrm{~J} / \mathrm{cm}^{2}$. Dark, solid, V-shaped curves are iso-energy gain lines with acceleration distance equal to dephasing length. Light dashed lines denote constant laser power. Light shaded area is delineated from the left by the line $P=P_{\text {crits }}$ from the right by $E_{i}=1 \mathrm{GeV} / \mathrm{m}$.

In another WG 6 discussion, the relative merits of self-injection, density downramp injection and colliding pulse injection were debated. Most laser acceleration experiments so far (e.g., [1-3]) have utilized self-injection, yielding one to several hundred $\mathrm{pC}$ charge and energy spread of 2 to 10 percent. Density down-ramp injection has the potential to produce much greater charge (up to hundreds of $\mathrm{nC}$, with $\sim 1 \%$ energy spread in simulations), but requires a ramp scale length less than a plasma wavelength for optimum injection. The ramp produced in the Taiwan experiments was somewhat longer, thus compromising injection efficiency. Production of sharper density down-ramps thus remains an important experimental challenge. Colliding-pulse injection has so far produced only $\sim 10 \mathrm{pC}$ of charge, but offers a high degree of control and flexibility. Several researchers pointed out that 3pulse and multi-color colliding pulse formats have yet to be explored. Possible approaches to increasing colliding-pulse injection efficiency include combining it with down-ramp injection and, as suggested by P. Michel (U. Nevada-Reno) using higherorder transverse injection laser modes.

In a joint discussion with WG 1 , there was widespread agreement that the holographic snapshots reported by N. Matlis (U. Texas) open an important new opportunity for benchmarking simulation codes. However, M. Downer (U. Texas), W. Mori (UCLA) and others cautioned that the snapshots were made in non-uniform 
gas jets, and thus averaged longitudinally over wakes of varying frequency and amplitude. For future experiments, the group recommended that single-shot wake measurements should be made in machined sharply-bounded uniform plasma profiles of systematically varied length. With such a "top-hat" profile, non-averaged snapshots could potentially be made and used to benchmark simulations with high fidelity. No significant barriers exist to applying the frequency-domain holography technique to charged-particle driven plasma wakes, where it could be used to characterize differences between electron-and positron-driven wakes. C. Geddes (LBNL) pointed out that the laser machining methods would also be valuable for sharpening and receding the plasma-vacuum exit boundary of a gas jet in order to improve short bunch-length characterization by CTR. Clearly combination of laser machining with advanced wake and bunch diagnostics will be an important focus of laser-plasma acceleration research in the near future.

A glance at Table 1 shows that future research will also rely heavily on petawatt lasers. We therefore invited 3 prominent laser design experts to summarize the prospects for this key enabling technology. E. Gaul (U. Texas) reviewed the status of the Texas Petawatt Laser. This $200 \mathrm{~J}, 150$ ps aystem utilizes existing technology, including optical parametric chirped pulse amplification in nonlinear crystals and power amplification in Nd:glass modules from the decommissioned NOVA laser at LLNL. This system is expected to be operational within one year. Its main limitation will be its "single shot" $(\sim 1$ pulse/hour $)$ mode of operation, imposed by the demands of heat removal from large, inefficiently pumped glass amplifiers. R. Sauerbrey (Forschungszentrum Rossendorf) reviewed longer-term plans for the Polaris laser at Jena, which incorporates significant new technology-efficient diode-pumped $\mathrm{Yb}$ :glass gain media-aimed at achieving higher repetition rate (e.g., $0.033 \mathrm{~Hz}$ at $1 \mathrm{pW}, 0.1 \mathrm{~Hz}$ at $0.1 \mathrm{PW}$ ). Finally, A. Galvanauskas (U. Michigan) reviewed scaling strategies for ultrashort fiber-based chirped-pulse amplification laser systems [26], including the prospects for reaching petawatt peak powers. Though the most speculative of the three approaches presented, fiber lasers offer the possibility of wallplug efficiencies $\sim 30 \%$ or higher and kilohertz repetition rate petawatt pulses if the technology can be scaled. The main technical challenges at present are increasing the duration of the chirped pulse from $\sim 10$ to $\sim 100 \mathrm{~ns}$ to avoid damage and self-focusing during amplification; increasing the core size of both solid and vacuum core fibers, while maintaining a single mode; coherently-phased wavelength multiplexing of the output of many smaller fiber laser systems. So far, as many as 6 fiber laser beams have been combined. Galvanauskas envisioned the possibility of coherently combining the output of as many as 1 million fiber amplifiers at an estimated cost of several megawatts electric power and $\$ 100 \mathrm{M}$ in order to achieve. Though these numbers are high, several experimentalists noted wryly that leading-edge simulation computers also require tens of megawatts of electrical power. While it is too early to speculate whether fiber laser technology can be scaled to this level, the future of laser-plasma acceleration will be tied closely to the success of laser designers in achieving efficient, high repetition rate petawatt lasers. 


\section{ACKNOWLEDGMENTS}

We thank the Workshop organizers and all of the participants in the Laser-Plasma Acceleration Working Group. We pay special tribute this year to Dave Sutter for his two decades of leadership and support for advanced accelerator research and his promotion of this workshop. We wish him well in retirement. In large part because of Dave's efforts over the years, support for this work was provided by the U.S. Department of Energy.

\section{REFERENCES}

1. S. M. Mangles et al., Nature 431, 535 (2004).

2. C. G. R. Geddes et al., Nature 431, 538 (2004).

3. J. Faure el al., Nature 431, 541 (2004).

4. T. Hosokai et al., Phys. Rev. Lett., in press (2006).

5. W. P. Leemans et al., "GeV electron beams from a centimetre-scale accelerator," Nature Phys, 2 , in press (2006).

6. D.J. Spence, S. M. Hooker, Phys. Rev. E 63,015401(R) (2001).

7. E. Esarey et al., Phys. Rev. Lett. 79, 2682 (1997):

8. G. Fubiani et al., Phys. Rev. E 70, 016402 (2004).

9. K. Nakamura et al., AIP Conf. Proc. 737, (Amer. Inst. Phys., NY, 2004), p. 901 (2004).

10. N. H. Matlis et al., "Shapshots of laser wakefields," Nature Phys., in press (2006).

11. S. P. LeBlanc et al., Opt. Lett. 25, 764 (2000).

12. K. Y. Kim et al., Appl. Phys. Lett. 81, 4124 (2002).

13. T. Watanabe et al., Nucl. Instrum. Methods A 480, 315 (2002).

14. W. P. Leemans et al., Phys. Rev. Lett. 91, 074802/1-4 (2003).

15. J. van Tilborg et al., Phys. Rev. Lett. 96, 014801 (2006).

16. C. G. Durfee et al., Phys. Rev. E 51, 2368 (1995).

17. P. Volfbeyn et al., Phys. Plasmas 6, 2269 (1999).

18. E. W. Gaul et al., Appl. Phys. Lett. 77, $4112(2000)$.

19. C.-H. Pai et al., Phys. Plasmas 12, 070707 (2005).

20. S. V. Bulanov et al., Phys. Rev. Lett. 78, 4205 (1997)

21. H. Suk et al., Phys. Rev. Lett. 86, 1011 (2001).

22. R. G. Hemker et al., Phys. Rev. ST Accel. Beams 5, 041301 (2002)

23. T.-Y. Chien et al., Phys. Rev. Lett. 94, 115003 (2005).

24. C.-H. Pai et al., Opt. Lett. 31, 984 (2006).

25. W. P. Leemans et al., IEEE Trans. Plasma Sci. 24, 331 (1996).

26. A. Galvanauskas, IEEE J. Sel. Top. Quant. Electron. 7, 504 (2001). 